Research Article

\title{
Knowledge, attitude and practice of pharmacovigilance among private healthcare professionals of Rajkot city
}

\author{
Bharti N. Karelia*, Kiran G. Piparava
}

Department of Pharmacology, P.D.U. Govt. Medical College, Rajkot-360001, Gujarat, India

Received: 30 October 2013

Revised: 10 November 2013

Accepted: 13 November 2013

*Correspondence to:

Dr. Bharti N. Karelia,

Email:

nirbharti_karelia@yahoo.co.in

(C) 2014 Karelia BN et al. This is an open-access article distributed under the terms of the Creative Commons Attribution Non-Commercial License, which permits unrestricted non-commercial use, distribution, and reproduction in any medium, provided the original work is properly cited.

\begin{abstract}
Background: Spontaneous reporting is an important tool in pharmacovigilance. However, its success depends on cooperative and motivated prescribers. In order to improve the reporting rate, it is essential to improve the Knowledge, Attitude and the Practices (KAP) of the healthcare professionals with regards to the ADR reporting and the pharmacovigilance. The present study was undertaken to evaluate the knowledge, attitude, and practices (KAP) of pharmacovigilance among private prescribers of Rajkot city.

Methods: It was a prospective questionnaire based survey type of study which was carried out among the private practitioners of Rajkot city. A questionnaire which was suitable for assessing the basic Knowledge, Attitude and the Practice (KAP) of pharmacovigilance was designed and distributed among the private doctors and filled up forms collected back and analyzed by microsoft excel 2007.

Results: Out of 600 distributed forms 332 forms were received back, reflecting a response rate of $55.33 \%$. $13.86 \%$ doctors didn't know about the term pharmacovigilance. $76.80 \%$ doctors experienced ADR during their clinical practices. $17.77 \%$ doctors maintain the record for patient safety and medico legal aspect.13.25\% doctors reported ADR at different places. Lack of knowledge and awareness and medico legal problems were common reasons for under reporting of ADR.78.30\% doctors agreed to keep ADR monitoring mandatory. Preferred methods for reporting ADR were electronic media and personal communication.

Conclusions: There was a great need to create awareness among the private doctors to improve the reporting of ADRs. Even though ADR reporting make compulsory by doctors unless there is no strict government rules and regulation success of pharmacovigilance programme is questionable.
\end{abstract}

Keywords: Adverse drug reaction, Knowledge, attitude, and practices study, Pharmacovigilance, Spontaneous reporting

\section{INTRODUCTION}

Effect and adverse effect are two sides of medicines. So not only to prescribe the right medicine for right disease but also not to produce extra harm to the patient is legal responsibility of every physician. Adverse drug reactions (ADR) are threat to the patient's safety and the quality of life and they increase the health care cost considerably. A chain of clusters of cases resulted due to use of some drugs (thalidomide disaster, sulfonamide disaster etc.) created an awareness of adverse drug reaction (ADR) in $18^{\text {th }}$ century and more and more attention is going to pay till date which resulted in emergence of a new science so called pharmacovigilance. ${ }^{1}$ Pharmacovigilance as defined by the World Health Organization (WHO) is 'the science and activities relating to the detection, assessment, understanding and prevention of adverse effects or any other possible drug-related problems. The
Pharmacovigilance Programme of India (PvPI) was initiated by the Government of India on 14.7.2010 with the All India Institute of Medical Sciences (AIIMS), New Delhi as the National Co-Ordination Centre for monitoring Adverse Drug Reactions (ADR) in the country for safe-guarding Public Health.The programme will be implemented in five phases. This year (year 20132014 ) it is in the $4^{\text {th }}$ phase. ${ }^{2}$ One of the short term goals of this programme is to enroll, initially; all MCI approved medical colleges in the program covering north, south, east and west of India. ${ }^{3}$ To fulfill this goal during initial phases of this programme different medical colleges are enrolled, that's why Pharmacovigilance science got place/identity in the civil hospitals and medical colleges but regards to private health care professional it is questionable. Its contribution to the Uppsala monitoring centre (UMC) database is very little. This is essentially due to the absence of a vibrant ADR monitoring system 
and also due to a lack of the reporting culture among the health care professionals. ${ }^{4}$ One of the important long term goal of this programme is to develop reporting culture amongst healthcare professionals and make ADR reporting mandatory for healthcare professionals. ${ }^{3}$

In order to improve the reporting rate, it is essential to improve the Knowledge, Attitude and the Practices (KAP) of the healthcare professionals with regards to the ADR reporting and the pharmacovigilance ${ }^{5}$. This study was a step which was taken in that direction and it endeavoured at evaluating the baseline KAP of the doctors in a private hospital, regarding the ADR monitoring and pharmacovigilance.

\section{METHODS}

It was a prospective questionnaire based survey type of study which was carried out among the private practitioners of Rajkot city. The study was approved by Institutional ethics committee. Practitioners with minimum graduation (MBBS, BDS) and all post graduates of allopathic medical science are included in the study. Homeopathic doctors, ayurvedic doctors, physiotherapist, radiologist, microbiologist and pathologists were excluded from the study. Those who did not willing to participate in the study were also excluded. A questionnaire which was suitable for assessing the basic Knowledge, Attitude and the Practice (KAP) of pharmacovigilance was designed and distributed among the doctors of private hospital of Rajkot city. Filled up forms collected back and analyzed by Microsoft excel 2007.

\section{RESULTS}

Out of 600 distributed forms 332 forms were received back, reflecting a response rate of $55.33 \%$. Among the doctors $84.63 \%$ were males while $15.36 \%$ were females with age ranges 22-72 years. Majority of participated doctors were postgraduate $(81.02 \%)$ followed by graduate (18.98\%). Among the postgraduate, respondent doctors have degree of doctor of medicine (MD) (46.68\%) followed by master of surgery (MS) $(28.91 \%)$, master of dental surgeon (MDS) (5.42\%). Among the MD specialist majority were physicians followed by obstetrics and gynecologist, pediatrician and anesthetists and dermatologist. Regarding MS majority doctors were general surgeons followed by orthopedics, ENT, ophthalmologist, neurosurgeons and spine surgeons.

Regarding knowledge of pharmacovigilance by health care professionals, out of 332 doctors46 (13.86\%) didn't know about the term pharmacovigilance. Among the 286 doctors as shown in table 2, majority had opinion that it is related to adverse effects $(46.15 \%)$ followed by awareness and study of ADR (30.41\%), detection and monitoring of ADR (10.48\%), drug/prescription survey (9.79\%) and reporting ADR (3.14\%) (Table 1$)$.

Table 1: Knowledge of pharmacovigilance by private healthcare professionals.

\begin{tabular}{|ll|}
\hline Opinions of doctors & No. of doctors $(\mathbf{n}=\mathbf{2 8 6})(\mathbf{\%})$ \\
\hline Side effect/ adverse effect of drug & $132(46.15)$ \\
\hline Awareness and study of adverse drug effect & $87(30.41)$ \\
\hline Detection and monitoring of ADR & $30(10.48)$ \\
\hline Drug /prescription survey & $28(9.79)$ \\
\hline Reporting of ADR & $9(3.14)$ \\
\hline
\end{tabular}

About $76.80 \%$ doctors agreed that they experienced ADR during their clinical practices. They managed ADR symptomatically and or stopping the drug. If it needed than patients were referred to higher center and did patient counseling. $82.22 \%$ doctors agreed that they did not keep any record of ADR in their hospital while $17.77 \%$ maintained the record and among them $13.55 \%$ mentioned the purpose. The most common purpose was patient's future safety $(9.33 \%)$, medico legal aspects (2.71\%) and for publication (1.5\%).86.14\% doctors never did reporting, $0.60 \%$ did not answer while $13.25 \%$ reported ADR and among them $10.24 \%$ mentioned the place where they reported ADR. They reported ADR at hospital level $(6.32 \%)$, pharmaceutical company $(2.11 \%)$ and at seminar $(1.81 \%)$.

Regarding attitudes towards underreporting, most common reason was lack of knowledge followed by lack of awareness, no established centre, fear of medico legal problem and relatives, lack of time and ignorance and negligence. The common drugs causing ADR faced by different consultants were antimicrobial followed by drugs acting on central nervous system, analgesic, drugs acting on gastrointestinal track, cardiovascular drugs. Very few ADR (1.80\%) were found to occur due to vaccine, blood and blood products, immunosuppressant and muscle relaxant. $78.30 \%$ of participated doctors agreed to keep ADR monitoring "Mandatory" and strongly believed that it will affect to patient safety directly or indirectly. $13.55 \%$ were unsure to keep this mandatory. There was also one spectrum $(6.92 \%)$ of respondent doctors who believed that reporting of ADR neither keep mandatory nor it will help to improve patients' safety (Table 2). 
Table 2: Knowledge of pharmacovigilance by private healthcare professionals.

\begin{tabular}{|ll|}
\hline Reasons for under reporting & No. of doctors \\
\hline Lack of knowledge of to whom and where report & 125 \\
\hline Lack of awareness/information about such reporting & 122 \\
\hline No any established reporting centre & 64 \\
\hline Fear of medicolegal problem and reaction from patient and relatives & 45 \\
\hline Lack of time \& busy schedule \& add extra burden & 30 \\
\hline Laziness, ignorance \& negligence & 22 \\
\hline Communication gap & 5 \\
\hline Only serious ADR should report & 5 \\
\hline Lack of guidelines & 13 \\
\hline No compensation & 1 \\
\hline No compulsion from the government & 10 \\
\hline
\end{tabular}

Regarding who should report ADR, most of the doctors agreed that medical healthcare professionals should report ADR followed by paramedical staff, patient himself and any person. Electronic media was the fastest way to search for any drug updates was suggested by almost all healthcare professions followed by medical text books, drug promotional literature, medical journals, MIMS, CIMS, conferences. As shown in table no.3 most common communicating media for reporting ADR as suggested by doctors was electronic media followed by post, telephone and meeting (Table 3).

Table 3: Knowledge of pharmacovigilance by private healthcare professionals.

\begin{tabular}{|ll|}
\hline Communicating media for ADR reporting & $*$ No. of doctors \\
\hline Electronic media(mail) & 225 \\
\hline By Post & 220 \\
\hline Direct contact through telephone & 157 \\
\hline Others (meeting, social networking site) & 10 \\
\hline *more than one option is selected by many doctors so total numbers is changed
\end{tabular}

\section{DISCUSSION}

Reporting of ADR is an essential component of pharmacovigilance and is crucial to the safety surveillance of marketed medicinal products. Spontaneous ADR reporting is a vital method for detecting new safety issues related to drugs. ADR contribute significantly to morbidity and mortality in clinical practice with its associated economic consequence. ${ }^{6}$ Many studies have evaluated the knowledge of healthcare professionals about pharmacovigilance. But reporting the same exclusively in private practicing doctors is reported in a very few studies.

Response rate reported in our study was lower (55.33\%) than that reported in other studies. Response rate of $61 \%$ was reported in another study carried out in prescribers. ${ }^{6}$ $13.86 \%$ doctors did not know about the term pharmacovigilance. $86.14 \%$ doctors had opinion that it is related to ADR. A study from India has reported encouraging findings regarding attitudes and perception of medical practitioners about pharmacovigilance. ${ }^{6}$ $76.80 \%$ doctors agreed that they experience ADR during their clinical practice and managed them by stopping the drug, giving symptomatic treatment and referring to higher centre. This was a positive reflection on the clinical skills and awareness about ADRs among the prescribers. However, the actual practice of reporting ADRs was different than that of the knowledge \& attitude exhibited by respondents.

Even as ADR reporting was considered to be important by a large majority of the respondents, the actual reporting was very low, just $13.25 \%$ of the respondents stated that they had reported an ADR previously. Similarly, one such study done in Mumbai by Hitesh Misra also cited similar findings of under-reporting of ADR to any of the national ADR monitoring centers $(2.9 \%)$ in spite of $90 \%$ of the respondents considering it is important. ${ }^{7}$ The reasons for reporting ADRs, as reported by Biriell and Edwards are, a desire to contribute to medical knowledge, identifying a previously unknown ADR, reactions to new drugs, and severity of the ADR. ${ }^{7}$ In our study too, the reasons for reporting, as cited by a majority of the respondents were, improving the safety of the patients, medicolegal aspects and for publication purpose. In this study, $13.25 \%$ doctors reported ADR and among them $10.24 \%$ mention the place where they report. 
$6.32 \%$ reported at hospital level, $2.11 \%$ at pharmaceutical company and $1.81 \%$ at seminar. Another study found same results that majority had reported to reporting centre followed by pharmaceutical company, conference and journal. $^{7}$

According to Inman, the reasons for under-reporting of ADRs can be complacency (belief that the serious ADRs are already documented when a drug is introduced in the market), diffidence (belief that reporting should be done when there is certainty that the reaction is caused by the use of a particular drug), financial incentives (rewards for reporting), ignorance (that only serious ADRs are to be reported), indifference (belief that a single report would make no difference), legal aspects (fear of litigation) and lethargy (excuses about lack of time or lack of interest). Some of these reasons were also documented in previous studies in India ${ }^{7}$ In our study a major reason observed was lack of knowledge and awareness. A majority of the doctors opined that the ADR reporting should be made compulsory $(71 \%)$ and some felt that it should be voluntary $(29 \%){ }^{6}$ in our study $78.30 \%$ of participated doctors agreed to keep ADR monitoring "Mandatory" and strongly believed that it will affect to patient safety directly or indirectly.

Spontaneous reporting of ADRs by patients and healthcare personnel, other than doctors, is practiced in many parts of the world. ${ }^{7}$ This was not recognized by the respondents in our study, as less than half of them identified nurses, pharmacists, and dentists to be capable of reporting ADRs. These findings were also observed in the study done in north India where respondents did not identify nurses and pharmacists as qualified reporters. ${ }^{7}$ This again indicates a lack of awareness of the principles and practice of pharmacovigilance among the respondents. Most common communicating media for reporting ADR suggested by doctors was electronic media. In another study same result were found that $50 \%$ of respondents suggest electronic option of ADR submission. ${ }^{8}$

\section{CONCLUSION}

Our study strongly suggested that there was a great need to create awareness among the private doctors to improve the reporting of ADRs. The training sessions must clarify the roles of the various healthcare professionals in pharmacovigilance. There should be closer relationship between the private doctors and the pharmacovigilance centers. The ADR reporting should be made an integral part of the clinical activities in order to improve the patient care. Even though ADR reporting make compulsory by doctors unless there is no strict government rules and regulation success of pharmacovigilance programme is questionable.

Funding: No funding sources

Conflict of interest: None declared

Ethical approval: Approval was taken from the Institutional Ethics Committee

\section{REFERENCES}

1. Gupta SK. Pharmacovigilance: current status \& future challenges. Indian Journal of Medical Specialities 2013;4(1):1-4.

2. Pharmacovigilance programme of India for assuring drug safety. Available at http://www.cdsco.nic.in/pharmaovigilance_intro.htm \#goal\&objectives. Accessed 1 October 2013.

3. NCC pharmacovigilance programme of India. Available at http://ipc.nic.in. Accessed 1 October 2013.

4. Gupta A, Uppal A. Adverse drug reaction reporting and pharmacovigilance. Knowledge attitude and practice among the resident doctors. J Pharma Sci 2011;3:1064-9.

5. Hardeep, Bajaj JK, and Rakesh K. A Survey on the Knowledge, Attitude and the Practice of Pharmacovigilance among the Health Care Professionals in a Teaching Hospital in Northern India. J Clin Diagno Res 2011 January; 7(1):97-99.

6. Pimpalkhute SA, Jaiswal KM, Suntakke SD, Bajait CS, Gaikwad A. Evaluation of a awareness about pharmacovigilance and adverse dryg reaction monitoring in resident doctors of a tertiary care teaching hospital. Indian J Med Sci 2012; 66:55-61.

7. Desai CK, Iyer G, Panchal J, Shah S, Dikshit RK. An evaluation of knowledge, attitude, and practice of adverse drug reaction reporting among prescribers at a tertiary care hospital. Perspect Clin Res. 2011 Oct;2(4):129-36.

8. Mishra H, Kumar V. Pharmacovigilance: Current Scenario in a Tertiary Care Teaching Medical College in North India. J Pharmacovigilance 2013;1:108. doi:10.4172/2329-6887.1000108.

doi:10.5455/2319-2003.ijbcp20140202

Cite this article as: Karelia BN, Piparava KG.

Knowledge, attitude and practice of

pharmacovigilance among private healthcare professionals of Rajkot city. Int J Basic Clin Pharmacol 2014;3:50-3. 